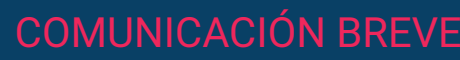

\title{
Seroprevalencia de COVID-19 en personas viviendo con VIH en el Área Metropolitana de Buenos Aires
}

\author{
Recibido: 7/10/21 Aceptado: 10/11/21
}

Yesica Longueira ${ }^{1,2}$, Florencia de la Fuente ${ }^{2}$, Fernando Montesano ${ }^{2}$, Horacio Salomón ${ }^{1,3}$, Gabriela Turk $^{1,3}$, María Florencia Quiroga ${ }^{1,3}$, Natalia Laufer ${ }^{1,3}$

\section{RESUMEN}

Se desconoce si las personas que viven con el $\mathrm{VIH}$ (PVV) tienen un mayor riesgo de contraer la infección por SARS-CoV-2. Los estudios serológicos prospectivos pueden permitir análisis de seroincidencia. Este estudio prospectivo incluyó a PVV sin antecedentes de vacunación contra SARS-CoV-2 de la Ciudad Autónoma de Buenos Aires (CABA) y Gran Buenos Aires (GBA) que concurrieron entre marzo y junio de 2021 a realizar sus controles al instituto INBIRS. La tasa de seroprevalencia de anticuerpos contra la proteína espiga del SARS-CoV-2 fue del 33,3\% (IC 24,9$42,5 \%$ ) para la población estudiada. Esta prevalencia fue significativamente mayor a la reportada previamente para la población general de la misma región geográfica. No se observó una asociación entre el recuento de linfocitos $T$ $\mathrm{CD}^{+}$con los niveles de IgG específica. En conclusión, el hallazgo de una alta seroprevalencia de anticuerpos contra SARS-CoV-2 entre las PVV en CABA y GBA puede sugerir una mayor susceptibilidad a la infección por este virus; sin embargo, puede ser también un marcador subrogante que indica la tasa de seroprevalencia en población general se encuentra subestimada.
${ }^{1}$ CONICET - Universidad de Buenos Aires. Instituto de Investigaciones Biomédicas en Retrovirus y SIDA (INBIRS). Buenos Aires, Argentina.

${ }^{2}$ Universidad de Buenos Aires, Facultad de Medicina. Buenos Aires, Argentina.

3 Universidad de Buenos Aires, Facultad de Medicina, Departamento de Microbiología, Parasitología e Inmunología. Buenos Aires, Argentina.

Autora para correspondencia: Dra. Natalia Laufer. Investigadora Adjunta CONICET.

Instituto de Investigaciones Biomédicas en Retrovirus y SIDA (UBA/CONICET)

Paraguay 2155 Piso 11, CABA - C1121ABG, Argentina. +54 1145083689 int 130

nlaufer@fmed.uba.ar

Los autores declaran que no tienen conflictos de interés para reportar.

Palabras clave: COVID-19, VIH, seroprevalencia. 


\section{Introducción}

La pandemia por SARS-CoV-2 lleva más de 18 meses de duración y todavía existen muchos interrogantes sobre la dinámica de la infección viral, entre ellos su comportamiento en diferentes grupos de individuos inmunosuprimidos, más aun entre las personas viviendo con VIH (PVV) y la posibilidad de sinergia entre ambas infecciones. Existen reportes con resultados contrapuestos en relación con la susceptibilidad, frecuencia y severidad que presenta la infección por SARS-CoV-2 en esta población (1-6). En algunas cohortes se han reportado prevalencias similares o menores que la población general (7-10). Las causas que podrían asociarse a diferentes tasas de prevalencia de infección entre las PVV son una mayor susceptibilidad asociada a la inmunosupresión, mayor conciencia y medidas de cuidado o la posible actividad de determinados antirretrovirales frente al SARS-CoV-2. Dado que la detección de la infección por SARS-CoV-2 mediante pruebas basadas en PCR puede subestimar la incidencia y prevalencia de casos (ya que en general no se realizan estudios a personas asintomáticas u oligosintomáticas), se necesitan estudios de seroprevalencia basados en la población para determinar con mayor precisión las tasas de ataque.

Nuestro objetivo fue determinar la seroprevalencia de anticuerpos contra SARS-CoV-2 en PVV con residencia en la Ciudad Autónoma de Buenos Aires y Gran Buenos Aires, que concurrieron a realizar sus controles de rutina al Instituto de Investigaciones Biomédicas en Retrovirus y SIDA (INBIRS).

\section{Materiales y métodos}

Estudio de corte transversal que se realizó entre el 9 de marzo y el 30 de junio de 2021. Se reclutaron 119 personas viviendo con VIH que cumpliesen con los siguientes criterios de inclusión: mayores de 18 años, que concurriesen a su control de rutina en el instituto INBIRS de la Ciudad Autónoma de Buenos Aires. Como criterios de exclusión se estableció la negativa a participar en el estudio y el haber recibido alguna de las vacunas para COVID-19. A los individuos se los invitó a donar una muestra de plasma para ser incluida en la colección HIV del Biobanco de Enfermedades Infecciosas, aquellos que accedieron dieron su consentimiento para ser incluidos. Se realizó una extracción de $3 \mathrm{ml}$ de sangre periférica. Se separó el suero y las muestras fueron estudiadas en tiempo real para determinar la presencia de anticuerpos de tipo IgG contra la proteína espiga (Spike) del SARSCoV-2. Se utilizó el ensayo COVIDAR (Fundación Instituto Leloir, Universidad de San Martín y Laboratorio Lemos S.R.L), ensayo inmunoenzimático, heterogéneo, no competitivo, basado en el método indirecto para la detección de anticuerpos IgG específicos contra el virus SARS-CoV-2.

Se recabaron datos demográficos, antecedentes de diagnóstico previo de COVID-19, tratamiento antirretroviral, carga viral de $\mathrm{VIH}$ y recuento de linfocitos T $\mathrm{CD}^{+}$ (determinados en la rutina de laboratorio efectuada en el INBIRS).

\section{Justificación del tamaño muestral}

El estudio fue diseñado para detectar la prevalencia de anticuerpos de isotipo IgG anti-SARS-CoV-2 en PVV. Para ello, se requería 100 individuos (asumiendo un alfa de 0.05 y un error beta de 0.2).

\section{Análisis estadístico}

Se analizaron con estadísticas descriptivas variables como sexo, edad, sintomatología, régimen antirretroviral, prevalencia de anticuerpos contra SARS-CoV-2 (número y proporción para variables discretas y media o mediana más desvío estándar o rango intercuartilo para variables continuas). Un valor $\mathrm{p}$ menor a 0.05 fue considerado estadísticamente significativo. Se utilizó una prueba no paramétrica binomial asintótica para comparar la prevalencia hallada con la de la población general.

\section{Resultados}

Se incluyeron 119 PVV, el 78\% de los individuos analizados correspondió al sexo masculino, con una media de edad de 41.7 años (SD 10.5), un 45\% refirió vivir en CABA y un 55\% en GBA. La media del recuento de linfocitos T CD4+ fue 621 células/ $\mu$ l (SD 286 células/ $\mu l)$, y el porcentual de linfocitos T CD4+ arrojó valores del $24,7 \%$ (SD 10,7\%). El 80\% de los individuos analizados presentó CV no detectable. Se pudieron recabar datos sobre el esquema antirretroviral (ARV) de 75 individuos. El $93 \%$ se encontraba recibiendo un esquema ARV basado en tenofovir y lamivudina o emtricitabina, $29 \%$ inhibidores de la integrasa, 32\% efavirenz, y un $36 \%$ inhibidores de la proteasa (ninguno de ellos recibía lopinavir). 
En 40 individuos se detectaron anticuerpos IgG específicos para SARS-CoV-2, lo que representa una seroprevalencia del 33,3\% (IC 24,9-42,5\%). De estas 40 PVV con serología reactiva, 19 individuos presentaban diagnóstico molecular previo de COVID-19 (47,5\%, IC $31,5-63,8 \%$ ), evidenciando que el $50 \%$ desconocía este antecedente (Figura 1). A los individuos con serología específica positiva y ausencia de diagnóstico previo de COVID-19 se les informó que podrían haber cursado la infección por SARS-CoV-2 en forma oligosintomática o asintomática.

Por otro lado, de los 79 individuos con resultados negativos de detección de IgG específica para SARSCoV-2, 2 presentaban diagnóstico molecular previo de COVID-19. En estos dos últimos casos se les explicó a los donantes que los motivos que pueden asociarse a un resultado negativo de serología en un individuo con diagnóstico molecular previo de COVID-19 pueden ser falta de sensibilidad del método utilizado para la medición de anticuerpos, ausencia de seroconversión que se observa en $5-15 \%$ de la población de individuos infectados (11) o en mucha menor frecuencia a un falso positivo de su diagnóstico molecular.
Del total de las 21 PVV con diagnóstico previo de COVID-19, se pudieron obtener datos sobre el cuadro clínico que presentaron en 8 casos; los síntomas más frecuentemente reportados fueron cefalea $(5 / 8,62 \%)$, anosmia $(4 / 8,50 \%)$, fiebre, odinofagia y disgeusia (todas en $3 / 8,37,5 \%$ ). Ningún caso cumplió criterios de gravedad ni requirió internación. No se encontraron diferencias en cuanto a la edad, el esquema ARV, ni tampoco en el nivel de linfocitos $T$ CD4 ${ }^{+}$de aquellos individuos que presentaron síntomas o cursaron la infección de modo asintomático. No se encontraron tampoco diferencias en cuanto al lugar de residencia (CABA o GBA) de los individuos en quienes se detectaron o no anticuerpos anti-SARS-CoV-2.

Al calcular la densidad óptica normalizada (NOD) como medida cualitativa de la presencia de anticuerpos antiSARS-CoV-2 (NOD = [ densidad óptica -OD- del ensayo OD cut off del ensayo]/OD control positivo), no se observó correlación entre los valores de NOD y el recuento de linfocitos $\mathrm{T} \mathrm{CD}^{+}$(Figura 2A). En coincidencia con observaciones previas relativas a la población general la edad mostró una correlación positiva con valores crecientes de NOD ( $r=0,39, p=0.018$, Figura 2B).

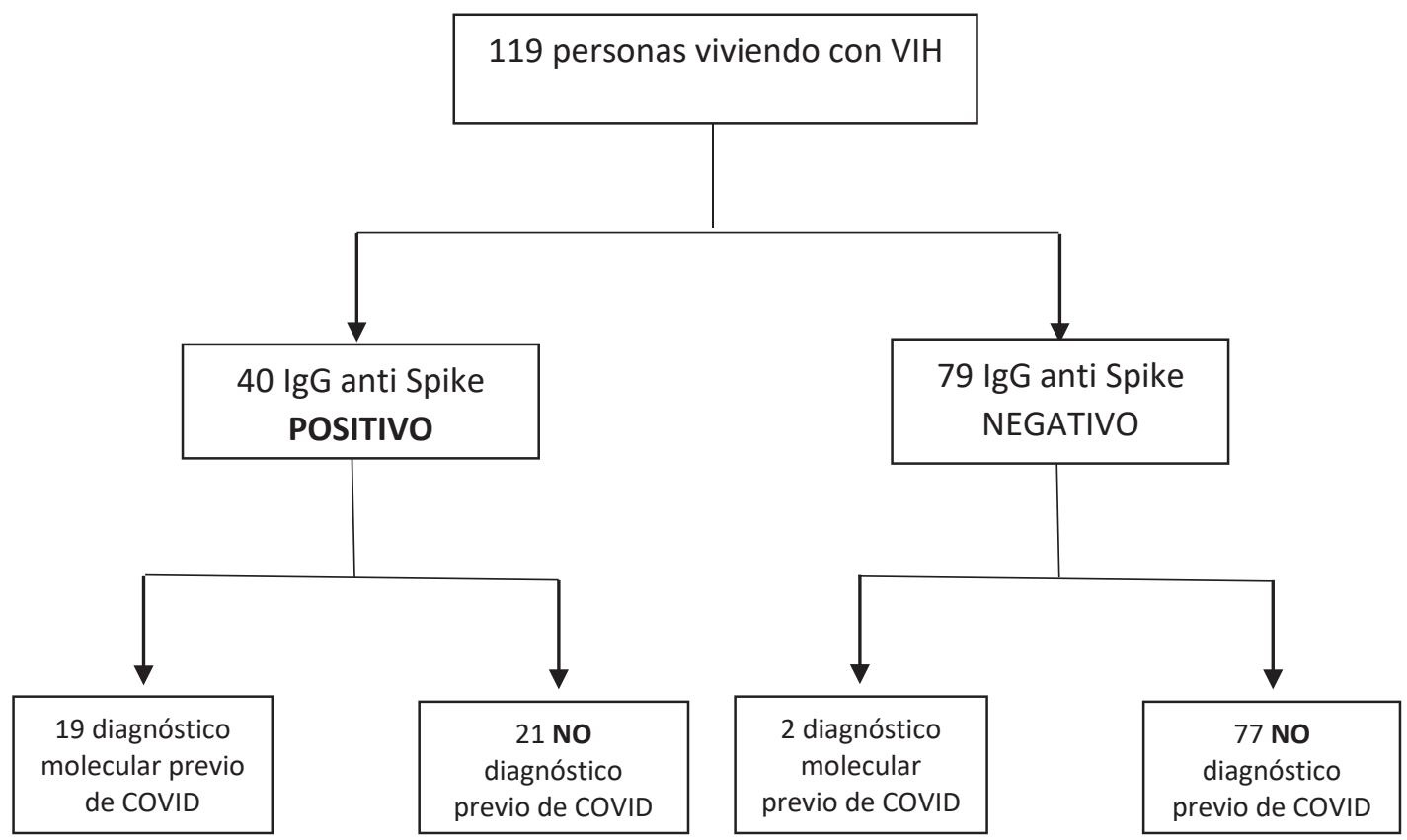


A.

CD4

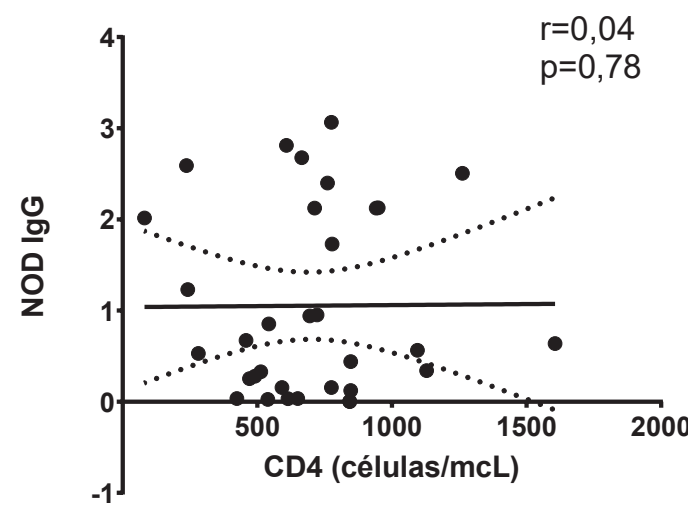

B.

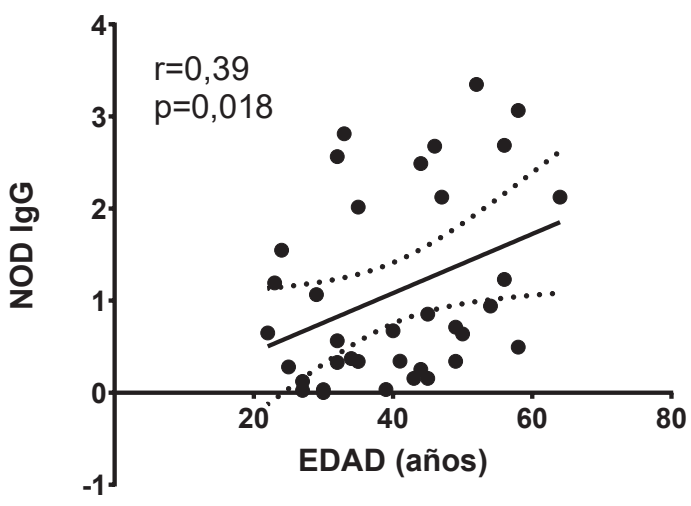

NOD: densidad óptica normalizada

\section{Discusión}

Este es el primer estudio que brinda una aproximación a la seroprevalencia de COVID-19 en PVV en Argentina y fue realizado durante el inicio de la segunda ola de casos de infección por SARS-CoV-2 en nuestro país. Entre las fortalezas de este trabajo debemos mencionar que resulta fundamental la investigación del impacto de la infección por SARS CoV-2 en poblaciones consideradas vulnerables, donde el grupo de PVV se ubica como prioritario. Esto es debido a la escasa información disponible respecto de la respuesta inmune antiviral en el marco de la inmunodeficiencia provocada por el $\mathrm{VIH}$. En este sentido, consideramos que el conocimiento generado por nuestro grupo aporta herramientas para el manejo del COVID-19 en el colectivo de PVV. Por otro lado, el número de individuos estudiados, el hecho de que la población estudiada pertenezca al AMBA y que los individuos participantes presenten una inmunidad conservada genera un sesgo en el estudio, mostrando así las limitaciones de este trabajo.

El grupo estudiado incluyó a PVV en su mayoría bajo tratamiento antirretroviral, con buena adherencia al mismo y que realizan controles periódicos. Las manifestaciones clínicas en esta población fueron leves o pasaron inadvertidas, pero, tal como se mencionó en las limitaciones del trabajo, estos datos deben ser interpretados con cautela y no extrapolarse a otras PVV, ya que no fue el objetivo de este estudio determinar las características del COVID-19, y se trata de un subgrupo bajo tratamiento y con un estatus inmunológico preservado.
La seroprevalencia obtenida en este grupo fue del $33,3 \%$, mientras que la reportada para la población general de CABA en diciembre 2020 en "Encuesta de Seroprevalencia de COVID-19, Ciudad de Buenos Aires" fue del 10\% (12). En marzo de 2021, los ministerios de Salud de la Provincia de Buenos Aires para la región del GBA y del GCBA para CABA reportaron una seroprevalencia promedio de $15 \%$ (13). Al comparar los valores de seroprevalencia para anticuerpos anti-SARS-CoV-2 en nuestra cohorte de individuos viviendo con $\mathrm{VIH}$ frente a los valores reportados en CABA y GBA, se observa una diferencia significativa; $p=0.00001$ (prueba no paramétrica binomial asintótica). Esta diferencia podría explicarse por: a) una mayor tasa de infección entre las personas viviendo con VIH frente a la población general; b) una subestimación de la seroprevalencia de la infección por SARS-CoV-2 en la población general y/o c) los diferentes momentos epidemiológicos en los que se realizaron los estudios de seroprevalencia, sumado a medidas más estrictas de aislamiento en el período que evaluó la prevalencia en población general con relación al período de este estudio. En particular, este último punto dificulta la interpretación de los resultados ya que resulta inapropiado realizar comparaciones entre momentos epidemiológicos diferentes.

Así, definir el peso de cada una de estas posibles causas en las diferencias observadas representa en sí mismo un importante motivo para profundizar este tipo de estudios en el escenario crítico de la actual pandemia por SARS-CoV-2, así como también resulta necesario seguir monitoreando si existen modificaciones en los datos conforme se modifica la dinámica de la pandemia. 


\section{Referencias}

1. Brown LB, Spinelli MA, Gandhi M. The interplay between HIV and COVID-19: summary of the data and responses to date. Curr Opin HIV AIDS. 2021;16(1):6373.

2. Sachdev D, Mara E, Hsu L, Scheer S, Rutherford G, Enanoria W, et al. COVID-19 Susceptibility and Outcomes Among People Living With HIV in San Francisco. J Acquir Immune Defic Syndr. 2021;86(1):19-21.

3. Chang JJ, Bruxvoort K, Chen LH, Akhavan B, Rodriguez J, Hechter RC. Brief Report: COVID-19 Testing, Characteristics, and Outcomes Among People Living With HIV in an Integrated Health System. J Acquir Immune Defic Syndr. 2021;88(1):1-5.

4. Huang J, Xie N, Hu X, Yan H, Ding J, Liu P, et al. Epidemiological, virological and serological features of COVID-19 cases in people living with HIV in Wuhan City: A population-based cohort study. Clin Infect Dis. 2020.

5. Xu Z, Zhang C, Wang FS. COVID-19 in people with HIV. The lancet HIV. 2020;7(8):e524-e6.

6. Inciarte A, Gonzalez-Cordon A, Rojas J, Torres B, de Lazzari E, de la Mora L, et al. Clinical characteristics, risk factors, and incidence of symptomatic coronavirus disease 2019 in a large cohort of adults living with HIV: a single-center, prospective observational study. AIDS. 2020;34(12):1775-80.

7. Naik SR, Kumar SS, Mittal A, Swain S, Ranjan S, Soneja M, et al. Seroprevalence of COVID-19 in HIV Population. 2021:2021.06.17.21259066.

8. Lombardi F, Ricci R, Belmonti S, Fabbiani M, Borghetti A, Baldin G, et al. Seroprevalence of SARS-CoV-2 Antibodies in HIV-Infected Patients in Rome, Italy during the COVID-19 Outbreak. Diagnostics (Basel). 2021;11(7).

9. Papalini C, Paciosi F, Schiaroli E, Pierucci S, Busti C, Bozza S, et al. Seroprevalence of anti-SARS-CoV2 Antibodies in Umbrian Persons Living with HIV. Mediterr J Hematol Infect Dis. 2020;12(1):e2020080.

10. Noe S, Schabaz F, Heldwein S, Mayer W, Ruecker K, Tiller FW, et al. HIV and SARS-CoV-2 co-infection: cross-sectional findings from a German 'hotspot'. Infection. 2021;49(2):313-20.

11. Longueira $\mathrm{Y}$, Polo $\mathrm{ML}$, InVi Vwg, Biobanco de Enfermedades Infecciosas Coleccion Cwg, Turk G, Laufer N. Dynamics of SARS-CoV-2-specific antibodies among COVID19 biobank donors in Argentina. Heliyon. 2021;7(10):e08140.
12. Encuesta de Seroprevalencia COVID-19. Metodología y resultados definitivos. Ciudad de Buenos Aires. Informe de Resultados N01509, 2020 https://www. estadisticaciudad.gob.ar/eyc/?p=115790.

13. Jait, A; Silva, A; Bolzán,A et al. Seroprevalencia de anticuerpos contra SARS-CoV-2 en barrios populares de la provincia de Buenos Aires. Diciembre 2020. Margen, Portal de Trabajo Social y Ciencias Sociales. https://www.margen.org/pandemia/index.html.. 


\section{COVID-19 Seroprevalence and PLHIV}

It is not known whether people living with HIV (PLHIV) are at increased risk of acquiring SARS-CoV-2 infection. Prospective serological studies can allow seroincidence analysis. This prospective study included PLHIV, without a history of vaccination against SARS-CoV-2, from the Autonomous City of Buenos Aires (CABA) and Buenos Aires surroundings (GBA), who attended INBIRS Institute between March and June 2021 to carry out their controls. The seroprevalence rate of antibodies against the SARSCoV-2 spike protein was $33.3 \%(\mathrm{Cl} 24.9-42.5 \%)$ for the study population. This prevalence was significantly higher than that previously reported for the general population of the same geographic region. No association was found between CD4+ T-cell counts with levels of SARSCoV-2 specific IgG. In conclusion, the finding of a high seroprevalence of antibodies against SARS-CoV-2 among PLHIV in CABA and GBA may suggest a greater susceptibility to infection; however, it can also be a surrogate marker that the seroprevalence rate in the general population is underestimated.

Keywords: COVID-19, HIV, seroprevalence. 